\title{
Spatiotemporal light localization in infiltrated waveguide arrays
}

Rasmussen, Per Dalgaard; Neshev, D.N-; Sukhorukov, A.A.; Krolikowski, Wieslaw; Bang, Ole; Lægsgaard, Jesper; Kivshar, Yu S.

Published in:

Joint Conference of the Opto-Electronics and Communications Conference and the Australian Conference on Optical Fibre Technology

Link to article, DOI:

10.1109/OECCACOFT.2008.4610643

Publication date:

2008

Document Version

Publisher's PDF, also known as Version of record

Link back to DTU Orbit

Citation (APA):

Rasmussen, P. D., Neshev, D. N., Sukhorukov, A. A., Krolikowski, W., Bang, O., Lægsgaard, J., \& Kivshar, Y. S. (2008). Spatiotemporal light localization in infiltrated waveguide arrays. In Joint Conference of the Opto-

Electronics and Communications Conference and the Australian Conference on Optical Fibre Technology (pp. 1. 2). IEEE. https://doi.org/10.1109/OECCACOFT.2008.4610643

\section{General rights}

Copyright and moral rights for the publications made accessible in the public portal are retained by the authors and/or other copyright owners and it is a condition of accessing publications that users recognise and abide by the legal requirements associated with these rights.

- Users may download and print one copy of any publication from the public portal for the purpose of private study or research.

- You may not further distribute the material or use it for any profit-making activity or commercial gain

- You may freely distribute the URL identifying the publication in the public portal 


\section{Spatiotemporal light localization in infiltrated waveguide arrays}

P.D. Rasmussen ${ }^{1,2}$, D.N. Neshev², A. A. Sukhorukov², W. Krolikowski ${ }^{2}$, O. Bang ${ }^{1}$, J. Lægsgaard ${ }^{1}$, and Yu. S. Kivshar ${ }^{2}$ ${ }^{1}$ DTU Photonics, Department of Photonics Engineering, Technical University of Denmark, 2800 Kgs. Lyngby Denmark

${ }^{2}$ Nonlinear Physics Centre and Laser Physics Centre, Centre for Ultrahigh bandwidth Devices for Optical Systems, Research School of Physical Sciences and Engineering, Australian National University, Canberra, ACT 0200, Australia Phone: +45 4525 6357, Fax: +45 4593 6581, Email: pdr@com.dtu.dk

\begin{abstract}
We study light propagation in hexagonal waveguide arrays and show that simultaneous spatiotemporal localisation is possible by combination of engineered anomalous dispersion through selective excitation of Bloch-modes and spatial confinement in a nonlinear defect mode.
\end{abstract}

\section{Introduction}

Nonlinear spatiotemporal light localisation, when light is confined in both space and time during propagation, is one of the enduring problems of nonlinear optics Predicted already in the early 90's [1] localization of light localised in three-dimensions (3D) (one time and two spatial dimensions) has proven to be a significant hurdle for experimental realisation. The reason for this is that in order to realise localisation in the form of a light bullet, one needs to simultaneously satisfy two important conditions: (i) to utilise a physical system with equal strengths of diffraction and dispersion (equal diffraction and dispersion lengths) and (ii) to employ a mechanism for stabilisation of the light bullets against collapse. A way to arrest collapse is to use a periodic structure [2]. In this work we present a novel realistic platform for realisation of spatiotemporal localisation based on liquid infiltrated cladding of photonic crystal fibres, and show its experimental feasibility.

Recent progress in photonic fabrication has made it possible to realise high quality periodic structures where both diffraction and dispersion can be simultaneously engineered. So far, however, studies of light propagation have only focussed on spatial effects. Nonlinear spatial localization in 1D and 2D periodic structures has been recently demonstrated [3]. But, to combine the spatial localisation with localisation in time, one need to also engineer anomalous or normal group velocity dispersion (GVD), depending on whether the nonlinearity of the material is focusing or defocusing respectively.

\section{Bloch modes on bandgap edges and defect states}

In our studies, we consider a waveguide array consisting of a hexagonal symmetry structure of high-index cylinders in a low-index background. Such a structure could for example be realized by infiltrating the holes in the cladding of a silica $\left(\mathrm{n}_{\mathrm{SiO} 2} \approx 1.45\right)$ photonic crystal fibre $(\mathrm{PCF})$ with nonlinear liquid $\mathrm{CS}_{2}\left(\mathrm{n}_{\mathrm{CS} 2} \approx 1.60\right)$. The distance between the centres of the high index inclusions of the structure is denoted $\Lambda$, and the hole diameter is denoted $d$ [Fig. 1]. For $d / \Lambda=0.50$ the range of allowed longitudinal propagation constants $(\beta)$ has a bandgap between the first and second band whenever $\lambda / \Lambda<1.15$, where $\lambda$ is the vacuum wavelength. $\mathrm{CS}_{2}$ has a large and fast focusing Kerr response $\left(\mathrm{n}_{2}=0.75 \cdot 10^{-18} \mathrm{~m}^{2} / \mathrm{W}\right.$ for $100 \mathrm{fs}$ pulses [4], approximately 30 times higher than silica), therefore a high intensity laser pulse can induce a nonlinear defect in the array. Such spatial localization of light is possible inside the bandgaps of the periodic structure. Bloch modes bounding the bottom of the spectral gaps experience normal diffraction, and therefore defect states bifurcating from these Bloch modes require a focusing nonlinearity. For defect states bifurcating from Bloch modes bounding the top of the gaps a defocusing nonlinearity is necessary, since these states experience anomalous diffraction.

In Fig. 1 we have shown the intensities and phases of the Bloch modes bounding the semi-infinite bandgap above the first band, and the bandgap between the first and the second band. Also in Fig. 1 we have shown the intensities of the corresponding defect states. The defect states are calculated by introducing a defect in the array of $\Delta \mathrm{n}_{\mathrm{NL}}=+10^{-3}$ for the top of the first and second band, and $\Delta \mathrm{n}_{\mathrm{NL}}=-10^{-3}$ for the bottom of the first band. The phase structure of the defect modes is similar to the phase of the Bloch modes. We see that the modes in the Top of first band
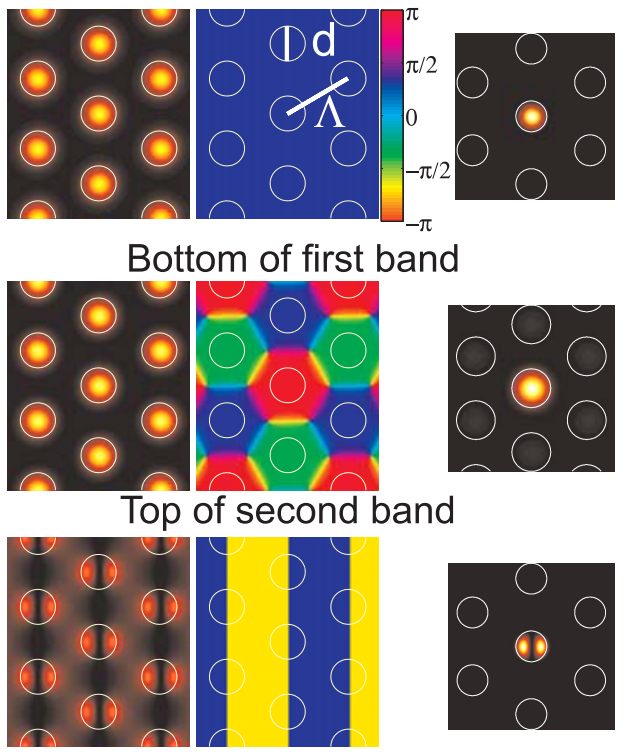

Fig 1. Intensities of Bloch modes at bandgap edges (left column), and their corresponding phases (middle column). Intensities of the defect states corresponding to the Bloch modes are shown in the right column. 
first band have $\mathrm{LP}_{01}$-like intensity structure. The top of the band has a flat phase structure, while the mode corresponding to the bottom of the band has $2 \pi / 3$ phase jumps between adjacent lattice sites. The top of the second band has a $\mathrm{LP}_{11}$-like intensity structure, with $\pi$ phase jumps going through the centres of the high index inclusions. Modes corresponding to both the first and second band have previously been excited experimentally in optically-induced photonic lattices [5].

\section{Group velocity dispersion of Bloch modes}

To find possibilities for existence of bright spatiotemporal solitons we search for conditions at which the defect states have anomalous GVD. The propagation constants of the defect states vary slightly from their corresponding Bloch modes, therefore we can calculate the GVD of the defect states by calculating the GVD of the corresponding Bloch modes. We define the GVD of the Bloch modes as $D=\omega^{2} /\left(2 \pi c v_{g}{ }^{2}\right) d v_{g} / d \omega$,

where $\omega$ is the angular frequency of the mode, $c$ is the speed of light in vacuum, and $v_{g}=(d \beta / d \omega)^{-1}$ is the group velocity of the mode with propagation constant $\beta(\omega)$. The material dispersion of the $\mathrm{CS}_{2}$ and the surrounding silica have also been included in the calculations.

In Fig. 2(a), solid lines we have plotted the GVD of the Bloch modes corresponding to the top of the first and second bands of a $\mathrm{CS}_{2}$ infiltrated array with $\Lambda=10 \mu \mathrm{m}$
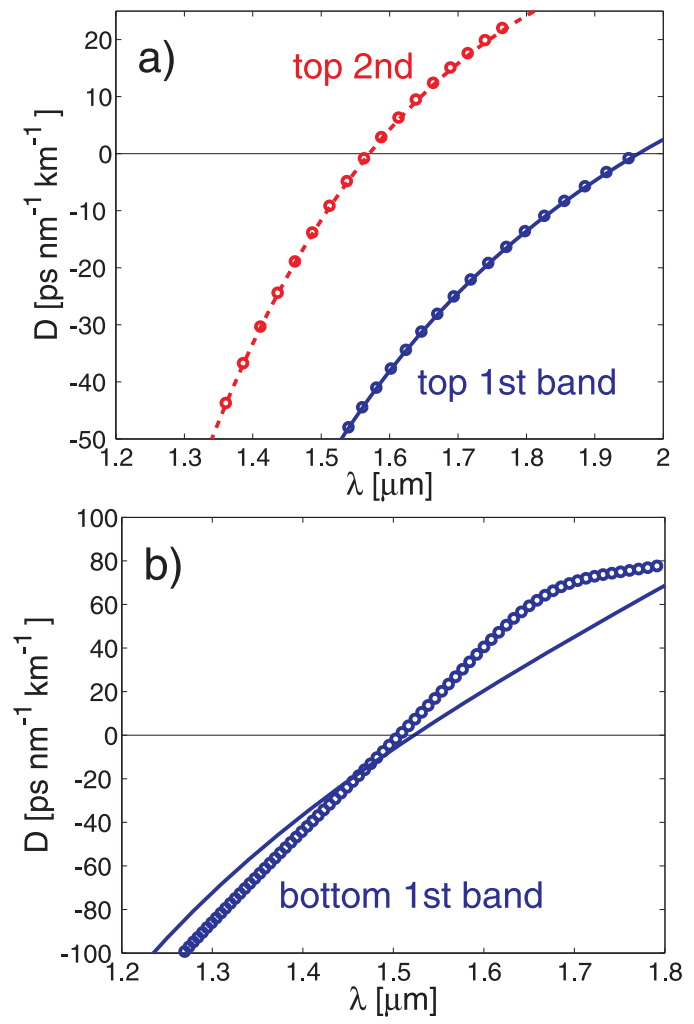

Fig. 2. Group velocity dispersion of Bloch modes (lines) and corresponding defect states (circles) of CS2 infiltrated waveguide arrays. (a) Dispersion of modes at the top of the first and second band for an array with $\Lambda=10 \mu \mathrm{m}$ and $d / \Lambda=0.45$. (b) Dispersion of mode at the bottom of the first band for an array with $\Lambda=3.5 \mu \mathrm{m}$ and $d / \Lambda=0.60$. and $d / \Lambda=0.45$. We also show (circles) the GVD of the defect mode corresponding to a defect of $\Delta \mathrm{n}_{\mathrm{NL}}=+10^{-3}$ at the central waveguide. We see that the GVD of the Bloch mode is indeed a good approximation to the dispersion of the defect mode. In Fig. 2(b) we have plotted the GVD of the Bloch mode corresponding to the bottom of the first band for an array with $\Lambda=3.5 \mu \mathrm{m}$ and $d / \Lambda=0.60$. Additionally, we also calculate the GVD of the defect mode corresponding to a defect of $\Delta \mathrm{n}_{\mathrm{NL}}=-10^{-3}$ at the central waveguide. We see that the GVD of the Bloch mode is again a good approximation to the dispersion of the defect mode, though larger deviations occur here than for the modes corresponding to the top of the first and second bands. We see that despite the strong normal material dispersion of $\mathrm{CS}_{2}$, regions of anomalous dispersion can be achieved for both focusing and defocusing nonlinearity. This opens a possibility of temporal localisation.

To achieve spatiotemporal localization with positive defect states (focusing nonlinearity), the fast Kerr response of the liquid should be responsible for both spatial and temporal localization. On the other hand, to employ negative defect states (defocusing nonlinearity), one needs to use a hybrid scheme. In this scheme two different types of nonlinearity need to be employed for compensation of pulse dispersion and beam diffraction, respectively. A defocusing type of nonlinearity, such as slow thermal nonlinearity, can be employed for spatial localization, while the self-focusing fast electronic response can be responsible for temporal localization. We note that in this case the fast nonlinearity should be weaker than the defocusing one, so the total induced defect will always remain negative. A negative defect can be induced by a thermo-optic effect, which arises when a small portion of the light is absorbed and heats the liquid. Since $\mathrm{CS}_{2}$ has a relatively large thermo-optic coefficient of $d n / d T=-7.9 \cdot 10^{-4} \mathrm{~K}^{-1}$, index changes of the order of $10^{-3}$ could be achieved even at small absorption levels. In this case the negative defect strength will depend not on the pulse peak power, but on the average beam power. Thus there will be two control parameters, which will contribute to the spatiotemporal localization: pulse peak power and average beam power. Further challenges associated with this scheme, including the spatially nonlocal aspect of thermal nonlinearity needs to be investigated.

\section{Conclusions}

We have proposed a new platform for demonstration of spatiotemporal light localization in 2D hexagonal periodic structures based on liquid infiltrated PCFs.

\section{References}

[1] Y. Silberberg, Opt. Lett. 15, (1990) p. 1282-1284.

[2] A. B. Aceves et al, Opt. Lett. 19, p. 329-331.

[3] D. N. Christodoulides et al, Nature 424 (2003), p. 817-823.

[4] I.P. Nikolakakos et al, IEEE J. Sel. Top. Quantum Electron. 10, p. 1164-1170.

[5] C. R. Rosberg et al, Opt. Lett. 32 (2007), p. 397-399. 